\title{
THEMED SECTION: MOLECULAR PHARMACOLOGY OF G PROTEIN-COUPLED RECEPTORS
}

\section{REVIEW}

\section{$\beta$-arrestins - scaffolds and signalling elements essential for WNT/Frizzled signalling pathways?}

\author{
Gunnar Schulte ${ }^{1}$, Alexandra Schambony² and Vítězslav Bryja ${ }^{3,4}$ \\ ${ }^{1}$ Section of Receptor Biology \& Signaling, Department of Physiology \& Pharmacology, Karolinska Institutet, Stockholm, Sweden, \\ ${ }^{2}$ Developmental Biology Unit, Biology Department, University of Erlangen-Nuremberg, Erlangen, Germany, ${ }^{3}$ Institute of \\ Experimental Biology, Faculty of Science, Masaryk University, Brno, Czech Republic, and ${ }^{4}$ Department of Cytokinetics, Institute \\ of Biophysics, Academy of Sciences of the Czech Republic, Brno, Czech Republic
}

$\beta$-arrestins were originally identified as negative regulators of $G$ protein-coupled receptor signalling. Recent studies have revealed that $\beta$-arrestins serve as intracellular scaffolds and signalling intermediates. Their diverse functions in intracellular signalling pathways provide mechanisms for achieving signal specificity that might be attacked for pharmacological intervention. Here, we summarize the importance of $\beta$-arrestin function for WNT [wingless (from Drosophila) and the oncogene int-1]/Frizzled (FZD) signalling. WNTs are secreted lipoglycoproteins that act through the seven transmembrane-spanning receptors of the FZD family. It recently became evident that $\beta$-arrestins are required for cellular communication by means of WNTs and FZDs both in cellular systems and in vivo. Although the overall importance of arrestin for WNT/FZD signalling remains obscure, interaction with the central phosphoprotein Dishevelled and the endocytic machinery implicates $\beta$-arrestin as a determinant of WNT signalling specificity, a mediator of WNT/FZD desensitization and a regulator of signalling compartmentation.

British Journal of Pharmacology (2010) 159, 1051-1058; doi:10.1111/j.1476-5381.2009.00466.x; published online 3 November 2009

This article is part of a themed section on Molecular Pharmacology of GPCR. To view the editorial for this themed section visit http://dx.doi.org/10.1111/j.1476-5381.2010.00695.x

Keywords: Dishevelled; RYK; ROR; $\beta$-catenin; canonical signalling; non-canonical signalling; endocytosis; desensitization; internalization; casein kinase

Abbreviations: AP-2, adaptor protein-2; CE, convergent extension; CK, casein kinase; DAAM1, dishevelled-associated activator of morphogenesis 1; DSH, Drosophila Dishevelled; DVL, Dishevelled; FZD $1-10$, Frizzled ${ }_{1-10} ;$ GPCR, G protein-coupled receptor; GRK2, G protein-coupled receptor kinase 2; GSK3, glycogen synthase kinase 3; JNK, C-JUN N-terminal kinase; MAPK, mitogen-activated protein kinase; MO, morpholinos; PCP, planar cell polarity; RAC-1, RHO, CDC42, RHO-like GTPases; RAP1, RAS-related protein 1, RAS proximate; ROR2, receptor tyrosine kinase ROR2; RYK, receptor tyrosine kinase RYK; TCF/LEF, T-cell factor/lymphoid enhancer factor; WNT, wingless (from Drosophila) and the oncogene int-1; XDSH, Xenopus Dishevelled

\section{Introduction}

$\beta$-arrestins were originally identified as negative regulators of G protein-coupled receptors (GPCR) mediating receptor desensitization, internalization, degradation and recycling (Dewire et al., 2007). The two isoforms of the family,

Correspondence: Gunnar Schulte, Section of Receptor Biology \& Signaling, Department of Physiology \& Pharmacology, Karolinska Institutet, S-171 77 Stockholm, Sweden. E-mail: gunnar.schulte@ki.se

Received 19 May 2009; revised 14 July 2009; accepted 21 July 2009 $\beta$-arrestin- 1 and 2 (also referred to as arrestin- 2 and 3), are highly homologous to the arrestins (arrestin-1 and 4) in the visual system that participate in the desensitization of rhodopsin.

$\beta$-arrestins were discovered in the late 1980s (Benovic et al., 1987; Lohse et al., 1990) as cofactors of GPCR kinase 2 (GRK2), with which they regulate the agonist-induced desensitization and internalization of $\beta_{2}$-adrenergic receptors to endosomes in a clathrin-dependent manner (Goodman et al., 1996). In subsequent years, a general mechanism evolved valid for many GPCRs that could explain both short-term and 
long-term desensitization as well as resensitization phenomena (Dewire et al., 2007). In brief, agonist stimulation of a GPCR results in receptor phosphorylation not only by serine/ threonine kinases, such as GRK2, but also by, for example, cAMP- or $\mathrm{Ca}^{2+}$-dependent protein kinases (PKA or PKC). The phosphorylated receptor serves as a docking site for cytosolic $\beta$-arrestin, which is therefore recruited to the membrane (Figure 1). Binding of $\beta$-arrestin to the receptor induces a high-affinity ternary complex consisting of the agonist-bound receptor and $\beta$-arrestin (Gurevich et al., 1997). Through direct interaction with clathrin and several adaptor proteins, such as adaptor protein-2 (AP-2), the $\beta$-arrestin-receptor complex is recruited to clathrin-coated pits. There the complex is internalized in a dynamin-dependent way resulting in a localization of the agonist- and $\beta$-arrestin-bound receptor to early endosomes. The affinity of $\beta$-arrestin to the receptor is then essential to determine subsequent events. Low affinity between receptor and $\beta$-arrestin (Class A receptors) will promote rapid recycling after internalization, whereas a highaffinity complex (Class B receptors) favours prolonged interaction and slow recycling (Lefkowitz and Shenoy, 2005).

With increasing understanding of the functional importance of $\beta$-arrestins in GPCR regulation, it became obvious that this scaffold protein not only mediates desensitization

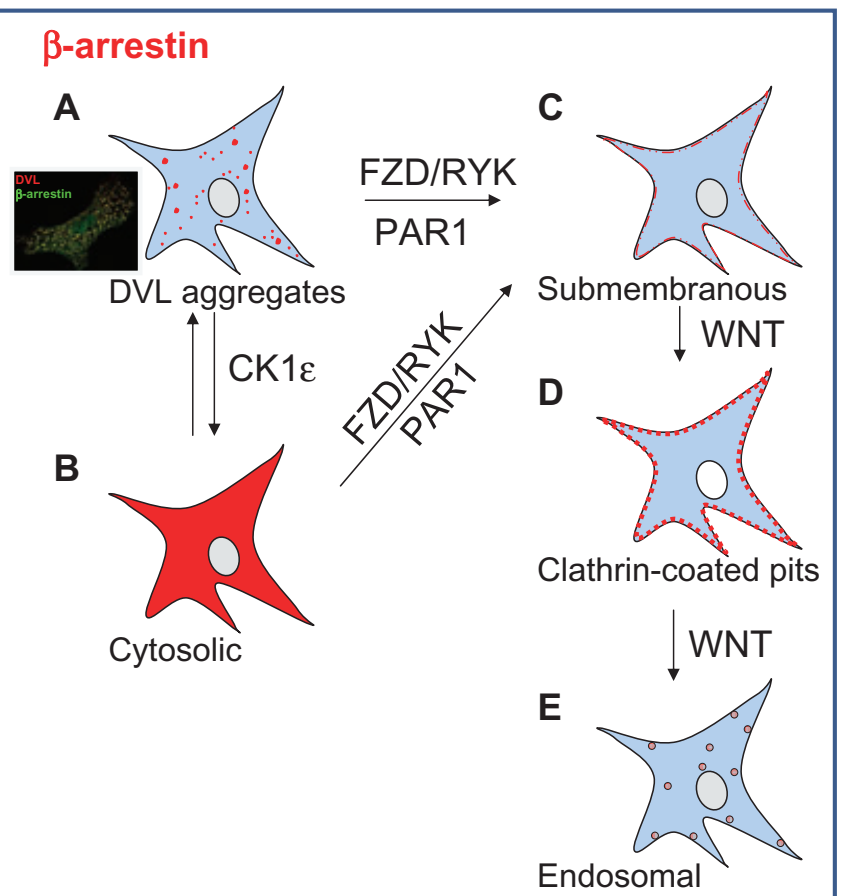

Figure 1 Schematic summary of possible localization of $\beta$-arrestin related to wingless (from Drosophila) and the oncogene int-1 (WNT) signalling in cells. In unstimulated cells, $\beta$-arrestin is evenly distributed in the cytosol. When Dishevelled (DVL) is present, it co-localizes with non-membraneous DVL aggregates (A). Upon phosphorylation of

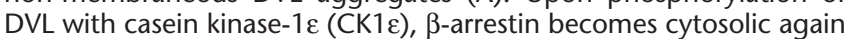
(B). In the presence of Frizzled (FZD), receptor tyrosine kinase RYK (RYK) and/or PAR1 $\beta$-arrestins are recruited to the plasma membrane (submembranous) (C) for further association with clathrin-coated pits upon ligand binding (D). Subsequently, the ligand-receptor- $\beta$ arrestin complex can be internalized through the endosomal pathway (E). The insert shows a mouse-embryonic fibroblast co-expressing $\beta$-arrestin-2 (green) and DVL-2 (red). and internalization, but also is responsible for many GPCRmediated signalling events. The first clue was the demonstration that $\beta$-arrestin interacts with the cytosolic tyrosine kinase c-SRC (Luttrell et al., 1999), indicating the possibility of a signalling event activated by GPCRs independently of heterotrimeric $G$ proteins. In the following years many $\beta$-arrestin interaction partners were identified (Xiao et al., 2007). Within a decade an entire series of signalling pathways active at so-called signalling endosomes had been identified. These pathways are based on the $\beta$-arrestin-receptor scaffold and the interaction of $\beta$-arrestins with a wide range of molecular entities: kinases, phosphatases, ubiquitin ligases, guanine nucleotide exchange factors and many more (Dewire et al., 2007; Lefkowitz, 2007; Xiao et al., 2007). The dual function of $\beta$-arrestins - their ability both to desensitize G proteindependent signalling and to support $G$ protein-independent signalling - offers fascinating possibilities for achieving specificity and signalling compartmentation in cells. However, it also presents challenges for attempts to develop drugs based on biased ligands that could promote or counteract one signalling branch over the other (Lefkowitz, 2007). Interestingly, the functional relevance of $\beta$-arrestin extends not only to many members of the GPCR family, but also to several nonGPCR receptors, including receptor tyrosine kinases (Hupfeld and Olefsky, 2007) such as insulin-like growth factor-1 (Girnita et al., 2005), receptor serine/threonine kinases such as transforming growth factor- $\beta$ receptors (Chen et al., 2003a) and NOTCH signalling (Mukherjee et al., 2005).

\section{Wingless (from Drosophila) and the oncogene int-1/Frizzled signalling}

Recently, a functional role of $\beta$-arrestins for WNT [wingless (from Drosophila) and the oncogene int-1]/Frizzled (FZD) signalling emerged. WNTs are secreted lipoglycoproteins (Nusse, 2003) that act in part through the seven transmembranespanning receptors of the FZD family of unconventional GPCRs (Schulte and Bryja, 2007). The signalling pathways downstream of FZD activation are complex, and their diversity is increased by the fact that 19 mammalian WNTs could at least theoretically activate 10 different $\mathrm{FZD}\left[\mathrm{FZD}_{1-10}\right.$; receptor nomenclature according to the International Union of Basic and Clinical Pharmacology (IUPHAR) and the BJP's Guide to Receptors and Channels (Alexander et al., 2008)]. So far, the selectivity of different WNTs for FZDs remains obscure (van Amerongen et al., 2008); only a few interaction profiles have so far been confirmed in mammals (see also FZD entry in the GPCR database of the IUPHAR at http://www.iuphar.org). Some of the main signalling routes that have been identified are reviewed extensively elsewhere (Polakis, 2000; Kohn and Moon, 2005; Nusse, 2005; Gordon and Nusse, 2006; Kikuchi et al., 2009) and summarized schematically (Macdonald et al., 2007; Semenov et al., 2007).

Briefly, WNT signalling pathways are divided into $\beta$-catenin-dependent and $\beta$-catenin-independent pathways. In the WNT/ $\beta$-catenin pathway, binding of WNT to FZD and a low-density lipoprotein-related protein5/6 (LRP5/6) co-receptor leads to the inhibition of a protein complex that 
includes glycogen synthase kinase 3 (GSK3). When active, this enzyme phosphorylates $\beta$-catenin, thus targeting it for proteasomal degradation. If the complex is inhibited, $\beta$-catenin is stabilized and can regulate target genes. $\beta$-catenin-independent pathways also act through FZD but involve different sets of effector proteins. These pathways can be subdivided into different branches according to their downstream effectors, such as WNT/RHO (Habas et al., 2003), WNT/RAC-1 (Habas et al., 2003) or WNT/RAS-related protein 1, RAS proximate (RAP1) (Tsai et al., 2007). Recently, the atypical receptor tyrosine kinase ROR2 (ROR2) has been identified as a WNT receptor that signals through a biochemically distinct $\beta$-catenin-independent pathway (Schambony and Wedlich, 2007).

\section{$\beta$-arrestin and FZD internalization}

The 10 FZD isoforms FZD ${ }_{1-10}$ and the closely related Smoothened (SMO) are classified as a separate family of GPCRs by the IUPHAR (Foord et al., 2005). Agonist-induced desensitization and internalization are a hallmark of classical GPCRs, and thus, it might be expected that similar mechanisms are valid for FZDs as well. So far, several studies have used tagged FZDs to investigate the mechanisms of WNT-induced and constitutive FZD internalization in various cell types (Chen et al., 2003b; Yamamoto et al., 2006; Bryja et al., 2007a). A breakthrough in the field was the study by Chen et al. (2003b), who provided evidence for a role of $\beta$-arrestin in the WNT-5Ainduced internalization of $\mathrm{FZD}_{4}$-green fluorescent protein (GFP) (Chen et al., 2003b). However, in contrast to the model for classical GPCRs, where $\beta$-arrestin binds the phosphorylated receptor directly, a phosphorylated adapter protein was required. It turned out that $\mathrm{Ca}^{2+}$-dependent protein kinasedependent phosphorylation of Dishevelled (DVL) was necessary to establish the FZD-DVL- $\beta$-arrestin complex, which is internalized in a clathrin-dependent manner (Figure 2). It was shown later that in addition to the interaction between DVL and $\beta$-arrestin, a presumably direct interaction between DVL and $\mu 2$ adaptin, a subunit of the AP- 2 complex, is required for $\mathrm{FZD}_{4}$ endocytosis (Yu et al., 2007). AP-2 binds DVL through a peptide YHEL motif localized C-terminal of the DEP (for DVL, EGL-10 and pleckstrin homology) domain and requires a lysine at position 446 (K446) within the DEP domain for optimal binding. The downstream signalling activated by WNT-5A, FZD 4 and PKC in HEK293 cells was not investigated in the initial study. However, mutations affecting K446 corresponding to K417 in Drosophila DVL - give rise to proteins that show reduced binding to AP-2 and are defective in planar cell polarity (PCP) signalling (Axelrod et al., 1998), indicating that $\beta$-arrestin and AP-2 mediated endocytosis are required in $\beta$-catenin-independent PCP signalling. This has been confirmed by two studies showing that $\beta$-arrestin is required for signalling to RHO-A (Kim and Han, 2007) and RAC-1 (Bryja et al., 2008). The role of $\beta$-arrestin for endocytotic activity in the PCP pathway was further emphasized by a recent study (Kim et al., 2008). Kim et al. showed that WNT-11 induced the formation of a $\mathrm{FZD}_{7} / \mathrm{DVL} / \mathrm{AP}-2$ complex that translocates to RAB5-positive endosomes in the Xenopus dorsal marginal zone in a $\beta$-arrestin-dependent manner. Sur-
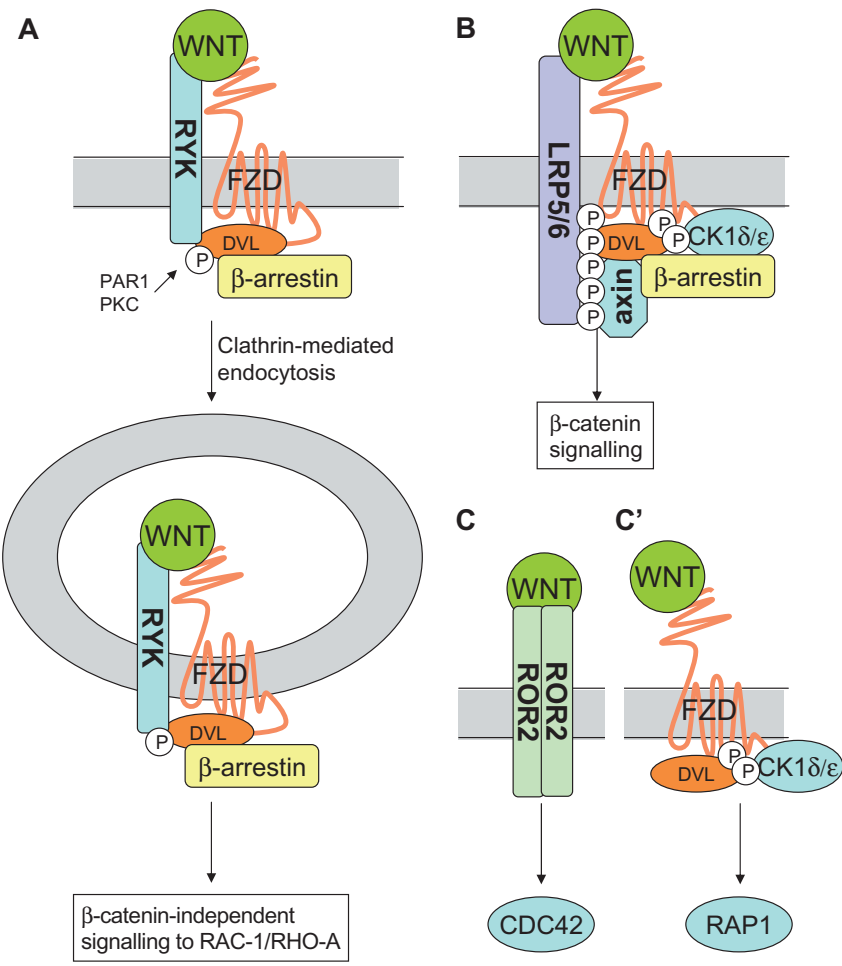

Figure 2 Emerging models for the role of $\beta$-arrestin in wingless (from Drosophila) and the oncogene int-1 (WNT) signalling. WNTinduced and Fizzled (FZD)-mediated signalling through $\beta$-catenindependent and -independent pathway requires $\beta$-arrestin and its interaction with Dishevelled (DVL). (A) In non-canonical WNT/RAC-1 and WNT/RHO pathways, recruitment of WNT/FZD/DVL complexes to clathrin-coated pits is mediated through $\beta$-arrestin interaction with adaptin/clathrin. Receptor tyrosine kinase RYK (RYK) is necessary for internalization and translocation of the complex to early endosomes. Translocation decreases cell surface numbers of FZDs and could contribute to desensitization of WNT/FZD signalling. In addition, signalling endosomes could contribute to specificity and compartmentation. (B) In the WNT/ $\beta$-catenin pathway, $\beta$-arrestin promotes WNT-induced phosphorylation of DVL and increases binding between axin and DVL. This probably happens in the context of the 'signalosome' derived from lipid rafts. It is not clear whether $\beta$-arrestin serves only as a scaffolding protein or whether $\beta$-arrestin-dependent endocytosis is required. (C) WNT-directed signalling through the atypical receptor tyrosine kinases of the ROR family, phosphatidylinositol-3'kinase, the small GTPase CDC42, c-JUN N-terminal kinase, paraxial protocadherin (PAPC) and subsequent modulation of convergent extension appears to be $\beta$-arrestin independent. $\left(C^{\prime}\right)$ Indirect experimental evidence suggests that the WNT/RAS-related protein 1, RAS proximate (RAP1) pathway involving casein kinase $1 \varepsilon(\mathrm{CK} 1 \varepsilon)$ could be $\beta$-arrestin independent. PAR1, partitioning defective mutant in $C$. elegans; PKC, protein kinase C; LRP5/6, lipoprotein-related protein5/6; ROR2, receptor tyrosine kinase ROR2.

prisingly, the dynamics of this complex was dependent on the presence of the receptor tyrosine kinase RYK (RYK), which was previously implicated in WNT signalling (Lu et al., 2004; Hendrickx and Leyns, 2008). In summary, these studies demonstrate that $\beta$-arrestin is essential for the formation of signalling endosomes in $\beta$-catenin-independent WNT signalling in vivo, and that this function is exerted by interactions between DVL, RYK and likely AP-2. In this context it should also be noted that the closely related SMO, a member of the FZD family (Foord et al., 2005), was shown to internalize in a $\beta$-arrestin and GRK2-dependent manner (Chen et al., 2004). 
The situation is more complex in $\mathrm{WNT} / \beta$-catenin signalling. Evidence that WNT/ $\beta$-catenin signalling also involves the formation of signalling endosomes arose from the findings that Drosophila wingless is internalized in vivo dependent on Arrow/ LRP and Drosophila $\mathrm{FZD}_{2}$ (Rives et al., 2006). In addition, a dominant-negative form of dynamin inhibited signalling to $\beta$-catenin in vitro (Blitzer and Nusse, 2006) and in vivo (Seto and Bellen, 2006). The observation that unspecific inhibitors of clathrin-mediated endocytosis such as monodansylcadaverine, chlorpromazine and hyperosmolaric sucrose blocked $\beta$-catenin signalling (Blitzer and Nusse, 2006) was initially interpreted as showing that clathrin-mediated and possibly $\beta$-arrestin-dependent endocytosis was required for $\mathrm{WNT} / \beta$ catenin signalling, similar to the $\beta$-catenin-independent pathways. Shortly after, however, we were surprised by the finding that inhibition of endocytosis by hyperosmolaric sucrose and $\mathrm{K}^{+}$depletion reduced protein levels of DVL and inhibited WNT-3A-induced $\beta$-catenin signalling (Bryja et al., 2007a), casting doubt on the original hypothesis.

Further, the co-receptor in the WNT/ $\beta$-catenin pathway, LRP6, is internalized after WNT-3A stimulation in a caveolindependent pathway (Yamamoto et al., 2006), and phosphorylation of LRP6 by casein kinase $1 \gamma(\mathrm{CK} 1 \gamma)$ and its association with caveolin in lipid rafts are required for $\beta$-cateninmediated signalling (Yamamoto et al., 2008). It is worth noting that no co-localization of LRP6 and CK1 $\gamma$ with early endosomes was observed in the latter study. However, constitutive internalization of $\mathrm{FZD}_{5}$ (Yamamoto et al., 2006) and Dickkopf (DKK1)-induced internalization of LRP6 (Yamamoto et al., 2008) are dependent on clathrin, and thus, potentially also on $\beta$-arrestin, indicating that clathrin-dependent endocytosis might also be involved in regulating the responsiveness of target cells to WNTs. RAB5, a small GTPase required for endocytic shuttling, has been found to act as a negative regulator of in vitro and in vivo signalling via wingless (the Drospophila homologue to WNT-1) (DasGupta et al., 2005; Rives et al., 2006). This finding supports the model in that, on the one hand, endocytosis of FZD and LRP5/6 contributes to limiting WNT responsiveness, and on the other hand, full activation of $\mathrm{WNT} / \beta$-catenin signalling is only achieved by formation of signalling endosomes. Currently, the role of $\beta$-arrestin in the internalization of receptor complexes of the canonical WNT pathway and the consequences for downstream signalling remain unclear. However, the diverging observations might be due to the different experimental systems employed, the FZD in question, the co-receptor involved, (Yamamoto et al., 2006) and possibly also, on the activating WNT. These issues are reviewed in detail by Kikuchi and Yamamoto (2007).

\section{$\beta$-arrestin and $\mathrm{WNT} / \beta$-catenin signalling beyond internalization}

Although the precise role of $\beta$-arrestin in FZD internalization and formation of signalling endosomes remains unclear, several studies have demonstrated that $\beta$-arrestin is necessary for $W N T / \beta$-catenin signalling. The first evidence for a functional involvement of $\beta$-arrestins in regulating $\mathrm{WNT} / \beta$ catenin signalling was provided by studying the effects of co-expression of DVL and $\beta$-arrestin on the transcriptional activity of T-cell factor (TCF)/lymphoid enhancer factor (LEF) (Chen et al., 2001). It was established that $\beta$-arrestin- 1 preferentially binds phosphorylated DVL-1 and 2, and that this enhances signalling capacity. These findings were later followed up by our groups, establishing $\beta$-arrestin as a necessary factor in WNT-3A-induced signalling to $\beta$-catenin and TCF/ LEF-mediated transcription. In vitro and in vivo analysis using both loss- and gain-of-function approaches revealed that DVL- $\beta$-arrestin interaction (see Figure 1 ) is required for WNT/ $\beta$-catenin signalling. Furthermore, immunoprecipitation experiments showed that $\beta$-arrestin participates in the formation of a ternary complex composed of phosphorylated DVL, $\beta$-arrestin and axin, implying that $\beta$-arrestin plays a functional role in the inactivation of the $\beta$-catenin destruction complex. Investigations in Xenopus laevis embryos then clearly supported the model of $\beta$-arrestin acting at the level of DVL, upstream of the stabilization of $\beta$-catenin, based on the inhibiting effects of $\beta$-arrestin morpholinos on axis duplication induced by Xenopus WNT-8, Drosophila Dishevelled $\Delta$ DEP (a $\beta$-catenin-dependent signalling-selective, constitutively active form of DVL lacking the DEP domain) - and CK1 $\varepsilon$ - but not $\beta$-catenin (Bryja et al., 2007b). This view suggesting a crucial role of $\beta$-arrestin in the destabilization of the destruction complex was recently supported by findings that $\beta$-arrestin binds axin, inhibits GSK3 phosphorylation and promotes TCF/LEF-dependent transcription in ovarian cancer cells (Rosanò et al., 2009).

\section{$\beta$-arrestin in $\beta$-catenin-independent WNT pathways}

As outlined above, $\beta$-arrestin is required for the WNT-induced internalization of FZD and RYK, and the regulation of PCP, which is essential for convergent extension (CE) movements in vertebrates (Kim and Han, 2007). Kim and Han showed that depletion of $\beta$-arrestin in Xenopus laevis embryos severely disrupted $\mathrm{CE}$, and that $\beta$-arrestin mediated the activation of the small GTPase RHO-A. Signalling through $\beta$-arrestin to regulate $\mathrm{CE}$ and the activation of RHO-A required interaction with DVL and the N-terminus of DVL-associated activator of morphogenesis 1 (DAAM1). This study described the role of $\beta$-arrestin for CE and RHO-A signalling in a DVL-dependent manner, and strongly implied that WNT-induced signalling along the same pathways would require $\beta$-arrestin in a similar manner.

Indeed, we recently reported that $\beta$-arrestin is required also for WNT-induced signalling via the small GTPase RAC-1 to elicit WNT-regulated CE (Bryja et al., 2008). Thus, $\beta$-arrestin appears to have an important scaffolding role in the WNT signalling pathways: it mediates the activation of small GTPases of the RHO family and brings important players such as DVL and DAAM1 in close proximity to provide signalling specificity in the regulation of CE.

\section{$\beta$-arrestin-independent WNT signalling}

CE of the mesoderm in vertebrate gastrulation is regulated by a complex network of signalling pathways. WNT/FZD 
signalling contributes to CE and is complemented by WNT/ ROR signalling (Schambony and Wedlich, 2007). It is still unclear whether WNT/ROR signals require FZDs or if ROR can act as an autonomous WNT receptor. Xenopus WNT-5A induces ROR signalling through phosphatidylinositol-3'kinase, the small GTPase CDC42, c-JUN N-terminal kinases and activating transcription factor 2 , which in turn regulates expression of paraxial protocadherin and thereby contributes to CE. While Xenopus WNT-11 function in CE in Xenopus laevis, which is mediated by a membranous FZD/RYK/DVL complex and the small GTPases RAC- 1 and RHO-A, is affected by expression levels of $\beta$-arrestin (Bryja et al., 2008; Kim et al., 2008), Xenopus WNT-5A, which acts via ROR2, is not (Bryja et al., 2008). Moreover, $\beta$-arrestin knock-down was fully rescued by constitutively active variants of the small GTPases RHO-A and RAC-1, but not by downstream effectors of Xenopus WNT-5A/ROR2 signalling, indicating that $\beta$-arrestin is a pathway-selective modulator of WNT signalling (Bryja et al., 2008).

Even though direct evidence is lacking, published data point to the WNT/CK1ع/RAP1 pathway (Tsai et al., 2007) as an additional $\beta$-arrestin-independent pathway (Figure 2C'). This assumption is based on several indications. Most importantly, CE defects induced by overexpression of CK1 $1 \varepsilon$ cannot be rescued by down-regulation of $\beta$-arrestin with morpholinos in Xenopus embryos (Bryja et al., 2008). In addition, formation of phosphorylated and shifted (PS)-DVL is elevated in unstimulated cells lacking $\beta$-arrestin-1/2. PS-DVL is formed as a consequence of CK1 $1 \varepsilon$ activity and is an expected intermediate of CK1 1 -driven $\beta$-catenin-independent pathway(s) (Cong et al., 2004; Klein etal., 2006; Strutt et al., 2006; Bryja et al., 2007c,d). It appears also that WNTs that do not activate $\beta$-catenin signalling (e.g. WNT-5A) are capable of inducing PS-DVL formation even in cells that lack $\beta$-arrestins, although the effects are severely retarded and less efficient than in the wild type cells (Bryja et al., 2007b; 2008). Moreover, overexpressed CK1 $1 \varepsilon$ can phosphorylate DVL even in the mouse embryonic fibroblasts lacking $\beta$-arrestins (V. Bryja and G. Schulte, unpublished). All these results argue that if a unique $\beta$-catenin-independent WNT pathway mediated by CK1ع/PSDVL/RAP1 does indeed exist, then $\beta$-arrestin is not part of its signalling cascade.

$\beta$-arrestin also does not seem to be involved in the $G$ protein-dependent WNT/Ca ${ }^{2+}$ pathway. Support for this conclusion comes from a study by Kim and Han (2007) that demonstrates that $\beta$-arrestin knock-down in Xenopus animal cap explants did not affect PK translocation, a hallmark of $\mathrm{WNT} / \mathrm{Ca}^{2+}$ pathway activation. However, as in the case of the WNT/CK1ع/RAP1 pathway, definitive proof showing whether $\beta$-arrestin is or is not required awaits further investigation.

\section{DVL - a crucial component for the effects of $\beta$-arrestin on WNT/FZD pathways}

Co-immunoprecipitation data demonstrate that $\beta$-arrestin strongly binds DVL, a crucial component of most WNT/FZD pathways (Chen et al., 2001; Bryja et al., 2007b; Kim and
Han, 2007; Kim et al., 2008). It has also been shown that $\beta$-arrestin can co-immunoprecipitate with overexpressed $\mathrm{FZD}_{2}, \mathrm{FZD}_{7}$ and RYK (Kim and Han, 2007; Kim et al., 2008) in HEK293 cells, suggesting the possibility of a direct interaction between FZD receptors and $\beta$-arrestin (Kim and Han, 2007). This direct interaction, however, has not yet received experimental support, and the current working model includes DVL to mediate the interaction of $\beta$-arrestin with membrane receptors such as FZD and RYK. Specifically, DVL knock-down can abolish FZD-induced re-localization of $\beta$-arrestin to the membrane (Kim and Han, 2007), and overexpression of DVL targeted to mitochondria can block RYKinduced membrane localization of $\beta$-arrestin (Kim et al., 2008). These data suggest that $\beta$-arrestin has a high affinity for DVL, which directs - at least in the context of WNT/FZD signalling - $\beta$-arrestin subcellular localization and subsequent function. So far, $\beta$-arrestin co-localized with DVL in all DVL-typical distribution patterns (Figure 1). DVL is usually localized in multiprotein aggregates visible as dots (Schwarz-Romond et al., 2005) (Figure 1A), which can be 'dissolved' by overexpression of CK1ع (Figure 1B) or stabilized by a kinase-dead CK1 or the CK1-inhibitor D4476 (Cong et al., 2004; Bryja et al., 2007d). $\beta$-arrestin, which is usually homogeneously distributed in the cytoplasm, follows the localization of DVL to 'dots', when DVL is overexpressed, and like DVL, it becomes more evenly distributed when both DVL and CK1ع are present (Bryja et al., 2007b). DVL can be recruited through the highly conserved KTxxxW sequence in the C-terminal tail of FZDs to the cell membrane (Wong et al., 2003; Punchihewa et al., 2009). This event can be mimicked by overexpression of PAR1 (partitioning defective mutant in C. elegans), a DVL kinase (Ossipova et al., 2005), or RYK (Kim et al., 2008). $\beta$-arrestin is not required for the translocation of DVL (Bryja et al., 2007b), but follows DVL to submembraneous compartments when FZD or RYK is present (Figure 1C) (Chen et al., 2003b; Kim et al., 2008). Membrane localization is also promoted by activating ligands (such as WNT-11) and inhibition of CK1 (Bryja et al., 2008). In cells rich in FZD and RYK, and with low CK1 activity, $\beta$-arrestin mediates WNT-induced internalization of membrane complexes containing FZD, RYK and DVL (Chen et al., 2003b; Kim et al., 2008). Such internalization and complex formation are required for activation of downstream components (e.g. RAC-1 or RHO-A) of the WNT/PCP pathway (Kim et al., 2008). In the context of the WNT/ $\beta$-catenin pathway, $\beta$-arrestin associates with CK1-phosphorylated DVL and axin, again following DVL and mediating downstream signalling events (Bryja et al., 2007b). In summary, DVL seems to act as an adaptor, bringing $\beta$-arrestin to the WNT machinery. $\beta$-arrestin in combination with CKs could thus be viewed as a scaffold that recruits additional specific effector proteins such as axin or DAAM1, depending on subcelluar localization and overall context. It remains to be shown whether this adaptor model indeed is generally applicable to all FZDs and other receptor components; however, the model suggests that DVL and $\beta$-arrestin in combination with specific kinases and effectors contribute to signalling specificity and balance the activity of the individual branches of WNT signalling. 


\section{$\beta$-arrestin as a link between WNT/FZD signalling and other signalling pathways}

In addition to linking $\beta$-catenin-dependent and several $\beta$-catenin-independent WNT pathways, $\beta$-arrestin provides also a connection to other signalling routes. This function can be indirect, as in the case of Kurtz (Drosophila $\beta$-arrestin)/ Deltex-mediated ubiquitinylation and degradation of NOTCH: $\beta$-arrestin interaction with NOTCH limits NOTCH signalling and, consequently, expression of wingless in the Drosophila wing disc (Roman et al., 2000; Mukherjee et al., 2005). An example of a more direct interface between $\beta$-arrestin and signalling pathway crosstalk was recently provided (Rosanò et al., 2009): in ovarian cancer cells, $\beta$-arrestin associates with the endothelin-A receptor $\left(\mathrm{ET}_{\mathrm{A}} \mathrm{R}\right)$, a Class 1 or rhodopsin-like GPCR, upon endothelin-1 (ET-1) stimulation. This is consistent with the classical model of $\beta$-arrestin function in GPCR signalling. Following ET-1 stimulation, the soluble tyrosine kinase SRC is recruited to the $\beta$-arrestin/ $\mathrm{ET}_{\mathrm{A}} \mathrm{R}$ complex, which induces $\beta$-arrestin-dependent SRC activation and transactivation of epidermal growth factor receptor (EGFR). EGFR activation results in elevated mitogen-activated PK (MAPK) signalling and, in addition, EGFR-dependent tyrosine phosphorylation of $\beta$-catenin. This stabilizing phosphorylation facilitates binding of $\beta$-catenin to TCF- 4 . In parallel, $\beta$-arrestin was shown to form a complex with $\mathrm{ET}_{\mathrm{A}} \mathrm{R}$ and axin that did not contain SRC, but inhibited GSK3 activity similar to the WNT/FZD-dependent signalling pathway. $\mathrm{ET}_{\mathrm{A}} \mathrm{R}$ is co-expressed with $\beta$-arrestin- 1 in advanced ovarian carcinoma, and ET-1 induced elevated $\beta$-catenin/TCF-mediated transcriptional activity and MAPK activity to promote invasiveness and metastasis in ovarian cancer xenografts. Taken together, $\beta$-arrestin could not only contribute to selectivity between different branches of WNT signalling, but also seems to contribute to crosstalk between WNT signalling, GPCRmediated signals and MAPK signalling.

\section{Emerging models}

In summary, several distinct models emerge from the data described here and show the crucial role of $\beta$-arrestins in many, but not all, branches of WNT signalling (Figure 2). $\beta$-arrestin supports the recruitment of receptor complexes to the endocytotic machinery and directs intracellular signalling to achieve signalling specificity and spatial compartmentation. Such endosome-driven signalling is controlled by $\beta$-arrestin in non-canonical WNT/FZD/RYK signalling to RAC-1, RHO-A and JNK (Figure 2A). In the WNT/ $\beta$-catenin pathway, the molecular details of $\beta$-arrestin action remain elusive. $\beta$-arrestin might be involved in clathrin-mediated FZD internalization with and without external WNT- and DKK1-induced LRP internalization, although there is so far no proof that $\beta$-arrestin participates in these processes. However, $\beta$-arrestin clearly promotes physical interaction of DVL and axin (Bryja et al., 2007b) (Figure 2B), which is required for signal transduction and has been shown to be required for $\mathrm{WNT} / \beta$-catenin signalling.

In addition, $\beta$-arrestin regulates WNT signalling and expression indirectly by affecting upstream pathways such as
NOTCH in the Drosophila wing (Mukherjee et al., 2005). $\beta$-arrestin has also recently been shown to mediate crosstalk between GPCR and $\beta$-catenin signalling (Rosanò et al., 2009). These diverse mechanisms of $\beta$-arrestin-mediated regulation of WNT signalling indicate on the one hand that $\beta$-arrestin acts as a scaffold that brings specific effector proteins together in the active signalling complex, and on the other hand that arrestin could provide a more general platform for signalling crosstalk and compartmentation. Working out the mechanisms that provide specificity towards one chain of downstream signalling events or the other will require further investigation.

It also remains to be determined whether the peculiar mechanisms of $\beta$-arrestin action in the WNT pathways, such as the requirement of DVL and RYK for FZD internalization, are applicable to all FZD isoforms, which co-receptors (such as RYK or LRP5/6) and intracellular interacting proteins (such as DAAM1 or CK1) contribute to pathway specificity, and how the interplay between desensitization of WNT signalling and formation of signalling endosomes is regulated. Last but not least, it will be exciting to see if the basic concepts of $\beta$-arrestin-mediated desensitization of heterotrimeric $G$ protein signalling will also hold true for the FZD family of unconventional GPCRs, and what role the $\beta$-arrestin-bindingpartner DVL might play in this context.

\section{Acknowledgements}

Work in our laboratories is funded by Karolinska Institutet (G.S.), the Swedish Research Council (K2008-68P-20810-01-4, K2008-68X-20805-01-4; G.S.), Jeansson Foundation (G.S.), the Foundations of the National Board of Health and Welfare of Sweden (G.S.), the Swedish Cancer Society (CAN2008/539; G.S.), the German Research Foundation (SCHA965 2-3 and SCHA965 6-1, A.S.), the EMBO Installation Grant (V.B.), the Czech Science Foundation (204/09/0498, 204/09/J030; V.B.), the Grant Agency of the Czech Academy of Sciences (KJB501630801; V.B.) and the Ministry of Education, Youth and Sports of the Czech Republic (MSM 0021622430, V.B.). Our collaboration is supported by an Institutional Grant for Younger Researchers from the Swedish Foundation for International Cooperation in Research and Higher Education.

\section{Conflict of interest}

The authors declare no conflict of interest.

\section{References}

Alexander SP, Mathie A, Peters JA (2008). Guide to receptors and channels (GRAC), 3rd edn. Br J Pharmacol 153 (Suppl. 2): S1-209.

van Amerongen R, Mikels A, Nusse R (2008). Alternative wnt signaling is initiated by distinct receptors. Sci Signal 1: re9.

Axelrod JD, Miller JR, Shulman JM, Moon RT, Perrimon N (1998). Differential recruitment of Dishevelled provides signaling specificity in the planar cell polarity and Wingless signaling pathways. Genes Dev 12: 2610-2622. 
Benovic JL, Kühn H, Weyand I, Codina J, Caron MG, Lefkowitz RJ (1987). Functional desensitization of the isolated beta-adrenergic receptor by the beta-adrenergic receptor kinase: potential role of an analog of the retinal protein arrestin (48-kDa protein). Proc Natl Acad Sci U S A 84: 8879-8882.

Blitzer JT, Nusse R (2006). A critical role for endocytosis in Wnt signaling. BMC Cell Biol 7: 28.

Bryja V, Cajánek L, Grahn A, Schulte G (2007a). Inhibition of endocytosis blocks Wnt signalling to beta-catenin by promoting dishevelled degradation. Acta Physiol (Oxf) 190: 55-61.

Bryja V, Gradl D, Schambony A, Arenas E, Schulte G (2007b). Betaarrestin is a necessary component of Wnt/beta-catenin signaling in vitro and in vivo. Proc Natl Acad Sci U S A 104: 6690-6695.

Bryja V, Schulte G, Arenas E (2007c). Wnt-3a utilizes a novel low dose and rapid pathway that does not require casein kinase 1-mediated phosphorylation of Dvl to activate beta-catenin. Cell Signal 19: 610-616.

Bryja V, Schulte G, Rawal N, Grahn A, Arenas E (2007d). Wnt-5a induces Dishevelled phosphorylation and dopaminergic differentiation via a CK1-dependent mechanism. J Cell Sci 120: 586-595.

Bryja V, Schambony A, Cajánek L, Dominguez I, Arenas E, Schulte G (2008). Beta-arrestin and casein kinase 1/2 define distinct branches of non-canonical WNT signalling pathways. EMBO Rep 9: 12441250 .

Chen W, Hu LA, Semenov MV, Yanagawa S, Kikuchi A, Lefkowitz RJ et al. (2001). beta-Arrestin1 modulates lymphoid enhancer factor transcriptional activity through interaction with phosphorylated dishevelled proteins. Proc Natl Acad Sci USA 98: 14889-14894.

Chen W, Kirkbride KC, How T, Nelson CD, Mo J, Frederick JP et al. (2003a). Beta-arrestin 2 mediates endocytosis of type III TGF-beta receptor and down-regulation of its signaling. Science 301: 13941397.

Chen W, ten Berge D, Brown J, Ahn S, Hu LA, Miller WE et al. (2003b). Dishevelled 2 recruits beta-arrestin 2 to mediate Wnt5A-stimulated endocytosis of Frizzled 4. Science 301: 1391-1394.

Chen W, Ren XR, Nelson CD, Barak LS, Chen JK, Beachy PA et al. (2004). Activity-dependent internalization of smoothened mediated by beta-arrestin 2 and GRK2. Science 306: 2257-2260.

Cong F, Schweizer L, Varmus H (2004). Casein kinase Iepsilon modulates the signaling specificities of dishevelled. Mol Cell Biol 24: 2000-2011.

DasGupta R, Kaykas A, Moon RT, Perrimon N (2005). Functional genomic analysis of the Wnt-wingless signaling pathway. Science 308: 826-833.

Dewire SM, Ahn S, Lefkowitz RJ, Shenoy SK (2007). Beta-arrestins and cell signaling. Annu Rev Physiol 69: 483-510.

Foord SM, Bonner TI, Neubig RR, Rosser EM, Pin JP, Davenport AP et al. (2005). International Union of Pharmacology. XLVI. G protein-coupled receptor list. Pharmacol Rev 57: 279-288.

Girnita L, Shenoy SK, Sehat B, Vasilcanu R, Girnita A, Lefkowitz RJ et al. (2005). \{beta\}-Arrestin is crucial for ubiquitination and downregulation of the insulin-like growth factor-1 receptor by acting as adaptor for the MDM2 E3 ligase. J Biol Chem 280: 24412-24419.

Goodman OB, Krupnick JG, Santini F, Gurevich VV, Penn RB, Gagnon AW et al. (1996). Beta-arrestin acts as a clathrin adaptor in endocytosis of the beta2-adrenergic receptor. Nature 383: 447-450.

Gordon MD, Nusse R (2006). Wnt signaling: multiple pathways, multiple receptors, and multiple transcription factors. J Biol Chem 281 : 22429-22433.

Gurevich VV, Pals-Rylaarsdam R, Benovic JL, Hosey MM, Onorato JJ (1997). Agonist-receptor-arrestin, an alternative ternary complex with high agonist affinity. J Biol Chem 272: 28849-28852.

Habas R, Dawid IB, He X (2003). Coactivation of Rac and Rho by Wnt/Frizzled signaling is required for vertebrate gastrulation. Genes Dev 17: 295-309.

Hendrickx M, Leyns L (2008). Non-conventional Frizzled ligands and Wnt receptors. Dev Growth Differ 50: 229-243.
Hupfeld CJ, Olefsky JM (2007). Regulation of receptor tyrosine kinase signaling by GRKs and beta-arrestins. Annu Rev Physiol 69: 561577

Kikuchi A, Yamamoto H (2007). Regulation of Wnt signaling by receptor-mediated endocytosis. J Biochem (Tokyo) 141: 443-451.

Kikuchi A, Yamamoto H, Sato A (2009). Selective activation mechanisms of Wnt signaling pathways. Trends Cell Biol 19: 119-129.

Kim GH, Han JK (2007). Essential role for beta-arrestin 2 in the regulation of Xenopus convergent extension movements. EMBO J 26: 2513-2526.

Kim GH, Her JH, Han JK (2008). Ryk cooperates with Frizzled 7 to promote Wnt11-mediated endocytosis and is essential for Xenopus laevis convergent extension movements. J Cell Biol 182: 1073-1082.

Klein TJ, Jenny A, Djiane A, Mlodzik M (2006). CKIepsilon/discs overgrown promotes both Wnt-Fz/beta-catenin and Fz/PCP signaling in Drosophila. Curr Biol 16: 1337-1343.

Kohn AD, Moon RT (2005). Wnt and calcium signaling: beta-cateninindependent pathways. Cell Calcium 38: 439-446.

Lefkowitz RJ (2007). Seven transmembrane receptors: something old, something new. Acta Physiol (Oxf) 190: 9-19.

Lefkowitz RJ, Shenoy SK (2005). Transduction of receptor signals by beta-arrestins. Science 308: 512-517.

Lohse MJ, Benovic JL, Codina J, Caron MG, Lefkowitz RJ (1990). beta-Arrestin: a protein that regulates beta-adrenergic receptor function. Science 248: 1547-1550.

Lu W, Yamamoto V, Ortega B, Baltimore D (2004). Mammalian Ryk is a Wnt coreceptor required for stimulation of neurite outgrowth. Cell 119: 97-108.

Luttrell LM, Ferguson SS, Daaka Y, Miller WE, Maudsley S, Della Rocca GJ et al. (1999). Beta-arrestin-dependent formation of beta2 adrenergic receptor-Src protein kinase complexes. Science 283: 655-661.

Macdonald BT, Semenov MV, He X (2007). SnapShot: Wnt/Betacatenin signaling. Cell 131: 1204

Mukherjee A, Veraksa A, Bauer A, Rosse C, Camonis J, ArtavanisTsakonas S (2005). Regulation of Notch signalling by non-visual beta-arrestin. Nat Cell Biol 7: 1191-1201.

Nusse R (2003). Wnts and Hedgehogs: lipid-modified proteins and similarities in signaling mechanisms at the cell surface. Development 130: 5297-5305.

Nusse R (2005). Wnt signaling in disease and in development. Cell Res 15: $28-32$.

Ossipova O, Dhawan S, Sokol S, Green JB (2005). Distinct PAR-1 proteins function in different branches of Wnt signaling during vertebrate development. Dev Cell 8: 829-841.

Polakis P (2000). Wnt signaling and cancer. Genes Dev 14: 1837-1851. Punchihewa C, Ferreira AM, Cassell R, Rodrigues P, Fujii N (2009). Sequence requirement and subtype specificity in the high-affinity interaction between human frizzled and dishevelled proteins. Protein Sci 18: 994-1002.

Rives AF, Rochlin KM, Wehrli M, Schwartz SL, DiNardo S (2006). Endocytic trafficking of Wingless and its receptors, Arrow and DFrizzled-2, in the Drosophila wing. Dev Biol 293: 268-283.

Roman G, He J, Davis RL (2000). Kurtz, a novel nonvisual arrestin, is an essential neural gene in Drosophila. Genetics 155: 1281-1295.

Rosanò L, Cianfrocca R, Masi S, Spinella F, Di Castro V, Biroccio A et al. (2009). Beta-arrestin links endothelin A receptor to beta-catenin signaling to induce ovarian cancer cell invasion and metastasis. Proc Natl Acad Sci U S A 106: 2806-2811.

Schambony A, Wedlich D (2007). Wnt-5A/Ror2 regulate expression of $\mathrm{XPAPC}$ through an alternative noncanonical signaling pathway. Dev Cell 12: 779-792.

Schulte G, Bryja V (2007). The Frizzled family of unconventional G-protein-coupled receptors. Trends Pharmacol Sci 28: 518-525.

Schwarz-Romond T, Merrifield C, Nichols BJ, Bienz M (2005). The Wnt signalling effector Dishevelled forms dynamic protein assemblies rather than stable associations with cytoplasmic vesicles. J Cell Sci 118: 5269-5277. 
Semenov MV, Habas R, Macdonald BT, He X (2007). SnapShot: noncanonical wnt signaling pathways. Cell 131: 1378.

Seto ES, Bellen HJ (2006). Internalization is required for proper Wingless signaling in Drosophila melanogaster. J Cell Biol 173: 95-106.

Strutt H, Price MA, Strutt D (2006). Planar polarity is positively regulated by casein kinase Iepsilon in Drosophila. Curr Biol 16: 13291336.

Tsai IC, Amack JD, Gao ZH, Band V, Yost HJ, Virshup DM (2007). A Wnt-CKIvarepsilon-Rap1 pathway regulates gastrulation by modulating SIPA1L1, a Rap GTPase activating protein. Dev Cell 12: 335347.

Wong HC, Bourdelas A, Krauss A, Lee HJ, Shao Y, Wu D et al. (2003). Direct binding of the PDZ domain of Dishevelled to a conserved internal sequence in the C-terminal region of Frizzled. Mol Cell 12: $1251-1260$.
Xiao K, McClatchy DB, Shukla AK, Zhao Y, Chen M, Shenoy SK et al. (2007). Functional specialization of beta-arrestin interactions revealed by proteomic analysis. Proc Natl Acad Sci U S A 104: 1201112016.

Yamamoto H, Komekado H, Kikuchi A (2006). Caveolin is necessary for Wnt-3a-dependent internalization of LRP6 and accumulation of beta-catenin. Dev Cell 11: 213-223.

Yamamoto H, Sakane H, Yamamoto H, Michiue T, Kikuchi A (2008). Wnt3a and Dkk1 regulate distinct internalization pathways of LRP6 to tune the activation of beta-catenin signaling. Dev Cell 15: $37-48$.

Yu A, Rual JF, Tamai K, Harada Y, Vidal M, He X et al. (2007). Association of Dishevelled with the clathrin AP-2 adaptor is required for Frizzled endocytosis and planar cell polarity signaling. Dev Cell 12: 129-141. 